\section{Human anti-rabbit antibodies}

S. Holdenrieder ${ }^{1}$ und P. Stieber ${ }^{2}$

${ }^{1}$ Institut für Laboratoriumsmedizin, Deutsches Herzzentrum München, Klinik an der Technischen Universität München, München, Deutschland

${ }^{2}$ Institut für Laboratoriumsmedizin, Klinikum der Universität München (LMU), München, Deutschland

\section{Synonym(e) HARA}

Englischer Begriff human anti-rabbit antibodies

Definition Heterophile Antikörper, die nach einer Applikation von Kaninchen-Immunglobulinen im Zuge von diagnostischen oder therapeutischen Maßnahmen gebildet werden.

Struktur $>$ Immunglobuline.

Funktion - Pathophysiologie Im Rahmen einer Immunszintigraphie oder einer Immuntherapie mit KaninchenImmunglobulinen werden heterophile Anti-Kaninchen-Antikörper gebildet, die in Testsysteme, in denen monoklonale Kaninchen-Antikörper verwendet werden, zu falsch positiven Werten führen können. Diese heterophilen Antikörper können auch bei Patienten, die mit „Frischzellen“ behandelt worden sind, vorkommen und falsch hohe Tumormarker- werte vortäuschen. Heterophile Antikörper werden nach intensivem Kontakt mit Tieren jedoch sehr selten auch bei Normalpersonen gefunden.

Untersuchungsmaterial- Entnahmebedingungen Serum.

Indikation Abklärung plötzlich erhöhter oder unerklärlich hoher Tumormarkerwerte ohne klinisches Korrelat und negativen HAMA-Werten.

Interpretation Heterophile Anti-Kaninchen-Antikörper sollten bei plötzlich erhöhten oder unerklärlich hohen Tumormarkerwerten ohne klinisches Korrelat und negativen HAMAWerten untersucht werden. Erhöhte HARA-Werte erübrigen eine weitere Diagnostik mit Tumormarkern.

Diagnostische Wertigkeit Abklärung plötzlich erhöhter oder unerklärlich hoher Tumormarkerwerte ohne klinisches Korrelat und negativen HAMA-Werten.

\section{Literatur}

Diamandis E, Fritsche HA, Lilja H (2002) Tumor markers. Physiology, pathobiology, technology, and clinical applications. AACC Press, Washington, DC

Stieber P, Heinemann V (2008) Sinnvoller Einsatz von Tumormarkern. J Lab Med 32:339-360 